\title{
Rôle de la flore fongique dans l'accumulation du calcium et du phosphore à la surface des fromages du type camembert (I)
}

\author{
par \\ M. METCHE et J. FANNI \\ Laboratoire de Biochimie Appliquée (E.N.S.A.I.A.) \\ 32, rue Sainte-Catherine - 54000 Nancy
}

\section{INTRODUCTION}

La participation de la flore fongique à l'affinage des pâtes molles moisies $(P$. caseicolum $)$ a mis en évidence l'importance des activités lipolytique et protéolytique de ce microorganisme $[1,2,3]$. Peu de chercheurs se sont intéressés à d'autres activités métaboliques [4], c'est pourquoi, dans ce contexte, nous nous sommes plus particulièrement intéressés à mettre en évidence une influence directe du développement de $P$. caseicolum sur la distribution des phosphates et du calcium à la surface des fromages. Ces éléments sont présents en quantités notables dans les fromages où ils contribuent à la fois à l'élaboration de la structure et de la flaveur.

Par ailleurs, il est bien établi à l'heure actuelle que les processus responsables de la pénétration et de l'accumulation des cations et des anions au niveau des membranes cellulaires des microorganismes relèvent de mécanismes bien définis que l'on peut ramener schématiquement à trois: diffusion simple, diffusion facilitée, processus biologiquement actifs [5, 6, 7, 8, 9]. La plupart du temps, le transport des cations bivalents s'effectue par l'intermédiaire d'un transporteur [10]. Par ailleurs, d'autres observations ont montré que des quantités non négligeables de calcium peuvent être fixées par les mitochondries des cellules rénales du rat au cours d'un processus nécessitant de l'ATP, des ions $\mathrm{Mg}^{++}$et des phosphates [11]. De même l'assimilation des ions orthophosphates chez la levure Saccharomyces cerevisiae dépend étroitement de la fourniture d'énergie métabolique aussi bien en aérobiose qu'en anaérobiose [9]. D'autre part, la pénétration de ces anions obéit à une cinétique de saturation, ce qui 
suggère ici encore l'intervention probable d'un récepteur, transporteur intermédiaire [12].

Le but de ce travail est de montrer ainsi, compte tenu des nombreuses observations relevées dans la littérature, que la moisissure Penicillium caseicolum participe activement à un processus de drainage et d'accumulation des ions $\mathrm{Ca}^{++}$et des phosphates, de l'intérieur vers la périphérie des fromages, et cela, indépendamment des processus simples de diffusion.

\section{MATERIELS ET METHODES}

\section{Souche de "Penicillium caseicolum"}

Utilisée pour les fabrications industrielles (Lacto-Labo, souche $\left.n^{\circ} 8473\right)$.

\section{Fromages du type Camembert}

Fabriqués au laboratoire à partir de lait pasteurisé contenant $27 \mathrm{~g} / \mathrm{l}$ de matière grasse. Après égouttage et salage en saumure, les fromages sont ensemencés par pulvérisation homogène d'une solution de spores dont la teneur est de $4.10^{7}$ spores/ml. L'affinage est conduit en hâloir à température et hygrométrie contrôlées $\left(12^{\circ} \mathrm{C}\right.$ et 92 p. $100 \mathrm{H}_{2} \mathrm{O}$ ).

Les échantillons de fromages prélevés sont soigneusement découpés en tranches horizontales de $3 \mathrm{~mm}$ d'épaisseur. Parallèlement, on effectue les mesures d'extrait sec et de matière grasse des fractions prélevées.

Un deuxième lot servant de témoin est badigeonné à l'aide d'une solution antifongique de Pimaricine (Gist-Brocades NV) $(10 \mu \mathrm{g} / \mathrm{ml})$ pour laisser la surface du fromage libre de toute flore fongique. Ce traitement a pour but de faire apparaître une différence d'accumulation en calcium et en phosphates dans les fromages suivant que le processus de transport est principalement de nature physicochimique (fromages dépourvus de flore fongique) ou biologique.

\section{Détermination du calcium et du phosphore}

Le calcium est dosé par spectrophotométrie d'absorption atomique (Appareil Jobin et Yvon, modèle Delta), tandis que le phosphore est déterminé selon la méthode de Bartlett [13]. Les résultats sont exprimés en fonction de l'extrait sec du fromage.

\section{Cultures en milieu liquide}

Nous utilisons un milieu à l'extrait de levures ( $5 \mathrm{~g} / 1)$ contenant $20 \mathrm{~g} / \mathrm{l}$ de glucose, ensemencé à raison de $1.10^{9}$ spores par litre. L'incu- 
bation est conduite pendant 70 heures en fermenteur de 2,5 1 (Biolafitte) à la température de $25^{\circ} \mathrm{C}$, sous agitation $(300 \mathrm{t} / \mathrm{mn})$ et insufflation d'air stérile $(1,5 \mathrm{l} / \mathrm{mn})$.

Le mycélium est ensuite récupéré par filtration sur papier stérilisé et lavé à l'eau distillée stérile.

\section{Etude de la cinétique de pénétration du calcium}

$100 \mathrm{~g}$ de mycélium humide, cultivé et recueilli selon $\S 4$, sont dispersés dans le milieu de Watson et Smith [4] dépourvu de calcium et de phosphore. Cette suspension est ensuite répartie dans 5 fermenteurs de $500 \mathrm{ml}$, thermostatés $\left(25^{\circ} \mathrm{C}\right)$ et agités $(300 \mathrm{t} / \mathrm{mn})$. Dans chaque fermenteur on introduit soit une quantité variable de $\mathrm{CaCl}_{2}$, $2 \mathrm{H}_{2} \mathrm{O}$ ou de $\mathrm{Ca}\left(\mathrm{NO}_{3}\right)_{2}(0,01$ à $0,1 \mathrm{M})$ pour une concentration fixe en $\mathrm{KH}_{2} \mathrm{PO} 4(0,025 \mathrm{M})$, soit une quantité variable en $\mathrm{KH}_{2} \mathrm{PO} 4(0,004$ à $0,04 \mathrm{M})$ pour une concentration fixée en $\mathrm{Ca}\left(\mathrm{No}_{3}\right)_{2}(0,01 \mathrm{M})$.

Pour certains essais, nous avons remplacé le glucose $(0,14 \mathrm{M})$ par des quantités stoéchiométriques de lactate de potassium ou de calcium. De même, pour certaines expériences, nous avons été amenés à substituer au $\mathrm{Ca}\left(\mathrm{No}_{3}\right)_{2}$ du $\mathrm{CaCl}_{2}$ afin de mettre en évidence le rôle de l'anion $\mathrm{Cl}^{-}$. Pour suivre l'accumulation du calcium et des phosphates dans le mycélium, on prélève des échantillons du milieu réactionnel toutes les 2 ou 3 minutes pendant $90 \mathrm{mn}$. Les quantités de calcium et de phosphates accumulées sont rapportées à l'unité de poids de mycélium sec correspondant.

\section{Cinétique d'incorporation du calcium en présence de phosphates}

$50 \mathrm{mg}$ de mycélium humide cultivé selon $\S 4$ sont introduits dans $50 \mathrm{ml}$ de milieu de Watson et Smith dépourvu de glucose, auquel on ajoute les réactifs :

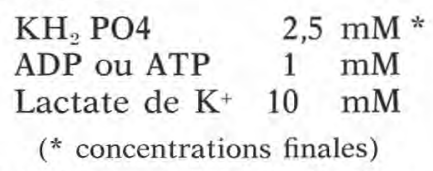

et $\mathrm{Ca}\left(\mathrm{NO}_{3}\right)_{2}$ dont on fait varier la concentration entre 0,025 et $0,4 \mathrm{M}$.

$\mathrm{Au}$ bout de $90 \mathrm{mn}$ d'incubation à $25^{\circ} \mathrm{C}$, on détermine la teneur en calcium du mycélium par spectrophotométrie d'absorption atomique. Les résultats sont exprimés en $\mu$ eq de $\mathrm{Ca}^{++} / 100 \mathrm{mg}$ de mycélium sec.

\section{Etude de la cinétique de pénétration des ions $\mathrm{H}_{2} \mathrm{PO}^{-}(\mathrm{Pi})$}

Pour étudier la cinétique de pénétration des ions $\mathrm{H}_{2} \mathrm{PO}^{-}$dans le mycélium en culture, nous avons suivi l'absorption de $\mathrm{KH}_{2}{ }^{32} \mathrm{PO} 4$ 0,5 à $5 \mathrm{mM}$ de radioactivité spécifique égale à $11,6 \mathrm{Ci} / \mu \mathrm{M}$. 
La méthodologie pour l'étude de la pénétration de ${ }^{32} \mathrm{Pi}$ est comparable à celle que Lin et Hanson [15] ont utilisée pour étudier la perméation des phosphates dans les racines de maïs.

Pour suivre l'accumulation de ${ }^{32} \mathrm{Pi}$, nous avons été amenés à opérer dans des tubes à essais contenant $10 \mathrm{mg}$ de mycélium humide que l'on incube pendant $90 \mathrm{mn}$ à $25^{\circ} \mathrm{C}$ en présence de ${ }^{32} \mathrm{Pi}(0,5$ à $5 \mathrm{mM})$, lactate de potassium $(10 \mathrm{mM}), \mathrm{Ca}\left(\mathrm{No}_{3}\right)_{2}(10 \mathrm{mM}$ ou $100 \mathrm{mM})$, avec ou sans ADP ou ATP $(1 \mathrm{mM})$, dans $10 \mathrm{ml}$ de milieu de Watson et Smith dépourvu de glucose, de calcium et de phosphore.

\section{RESULTATS ET DISCUSSION}

\section{Distribution du calcium et du phosphore dans un fromage en cours d'affinage}

Nous avons étudié la distribution du calcium et du phosphore dans des fromages du type Camembert de dimensions courantes provenant d'une même fabrication, en fonction du temps d'affinage (12 j). L'étude a porté sur des prélèvements effectués à des niveaux différents des fromages :

- la partie interne, au voisinage du centre de gravité ;

- la partie externe (croûte), au niveau des deux faces. Chaque point est la moyenne de 8 déterminations.

Les résultats reportés figure 1, exprimés en meq/100 g d'extrait sec permettent de dégager immédiatement une conclusion :

Tandis que dans la partie interne du fromage les teneurs en calcium et en Pi varient peu, il n'en est pas de même dans la croûte. Pendant les 4 premiers jours d'affinage, les teneurs en calcium et en $\mathrm{Pi}$ évoluent de la même manière que pour la partie interne, puis on assiste à un accroissement significatif qui tend à se stabiliser au bout de 12 jours. Le processus est particulièrement accusé pour le calcium.

Il est à noter par ailleurs que ce processus d'accumulation au niveau de la croûte du fromage coïncide exactement avec la période de croissance de $P$. caseicolum, depuis la phase de latence qui s'étale sur 4 jours dans les conditions de l'affinage, jusqu'à la phase stationnaire que l'on atteint aux environs du $10^{e}$ jour. Sans exclure pour autant les processus physico-chimiques de diffusion et d'échange de $\mathrm{Na}^{+}$et $\mathrm{Cl}^{-}$qui tendent à pénétrer vers l'intérieur du fromage, il apparaît comme hautement probable que le développement de la flore superficielle participe à ce mécanisme. On peut penser notamment que le développement de la flore fongique contribue directement au drainage des phosphates et du calcium, de l'intérieur vers la partie périphérique des fromages. 


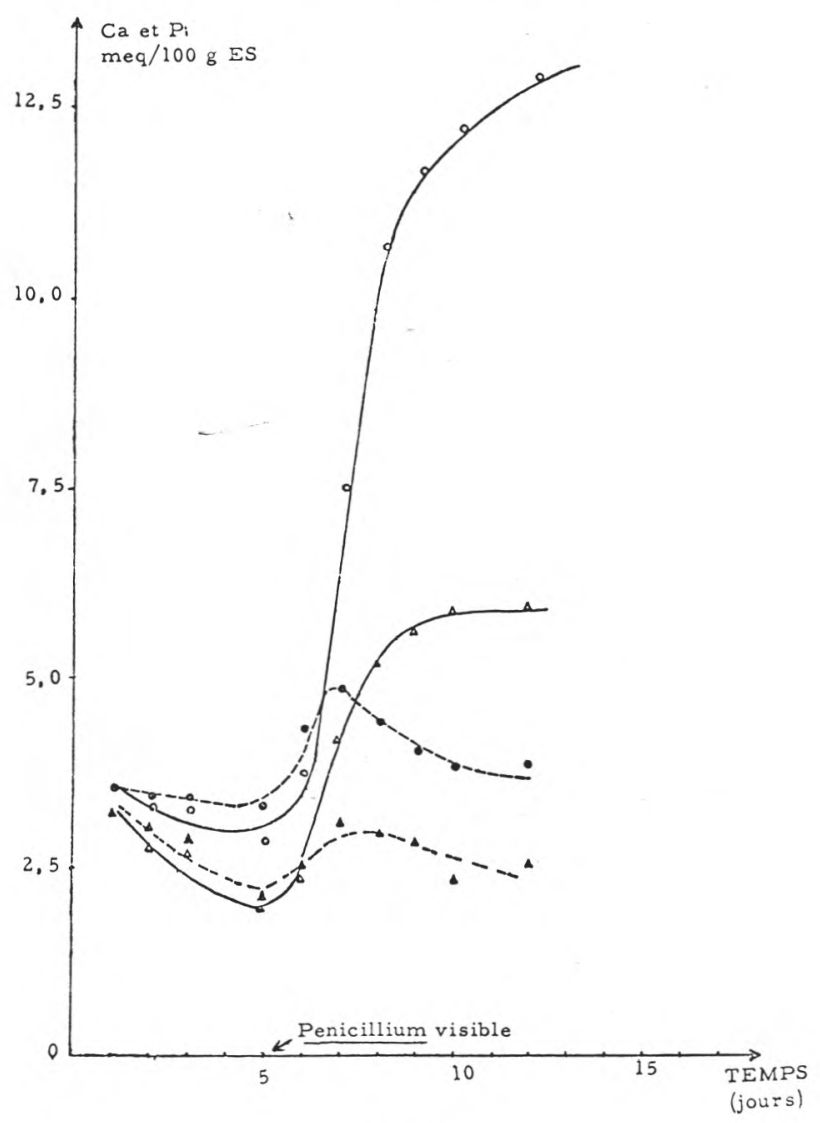

fig. 1

Etude de la variation des teneurs en calcium et en

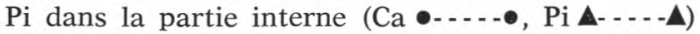
et dans la partie externe des fromages ( $\mathrm{Ca} \mathrm{O-}-.-\mathrm{O}$, $\operatorname{Pi} \triangle \ldots-. \triangle$ ). La flèche indique le jour où la moisissure est visible à l'œil nu.

Parallèlement et a priori, on aurait pu penser que l'étude de la distribution du rapport $(\mathrm{Ca}) /(\mathrm{Pi})$ à l'intérieur et à l'extérieur $\mathrm{du}$ fromage, permettrait de préciser la nature des combinaisons calciumphosphates qui participent au déplacement du calcium et du phosphore.

Les valeurs des différents rapports $(\mathrm{Ca}) /(\mathrm{Pi})$ sont rassemblées figure 2. Tandis que le rapport $(\mathrm{Ca}) /(\mathrm{Pi})$ pour l'intérieur du fromage diminue de manière continue, passant de 1,51 en début d'affinage à 


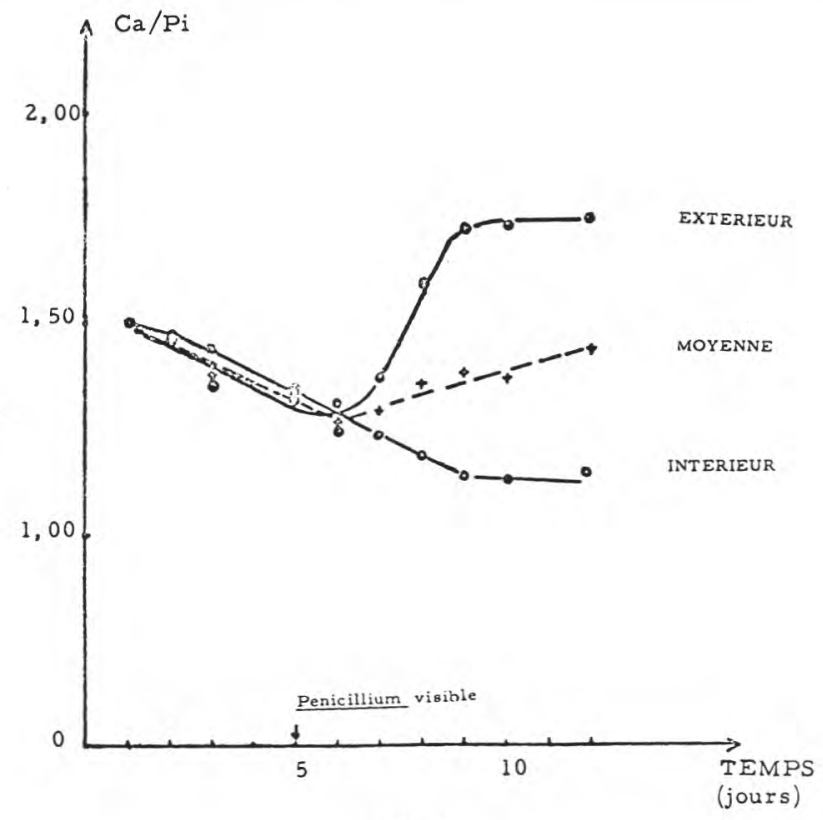

fig. 2

Variation $\mathrm{du}$ rapport $\mathrm{Ca} / \mathrm{Pi}$ dans les fromages en cours d'affinage. Les valeurs obtenues se rapportent respectivement à la fraction externe ou croûte $(\bullet-\bullet)$, à la fraction interne $(\mathrm{O}-\mathrm{O})$ et à la moyenne correspondant à l'ensemble du fromage $+\cdot \cdot \cdot+$ ).

1,15 au bout de 12 jours, au niveau de la croûte on assiste après 5 jours d'une évolution comparable, à un accroissement significatif. Ainsi, $(\mathrm{Ca}) /(\mathrm{Pi})$ atteint respectivement 1,35 après 5 jours, 1,60 après 8 jours et 1,75 le $12^{\circ}$ jour. La moyenne de ces résultats pour l'ensemble du fromage fait apparaître un rapport $(\mathrm{Ca}) /(\mathrm{Pi})$ de 1,38.

L'accroissement du rapport $(\mathrm{Ca}) /(\mathrm{Pi})$ dans la croûte après 5 jours peut s'expliquer par une distribution du calcium entre diverses formes salines comportant comme anions de compensation des ions inorganiques (entre autres phosphates) et des ions organiques, notamment l'ion lactate. L'engagement $\mathrm{du} \mathrm{Ca}^{++}$sous forme de combinaisons solubles assimilables par le mycélium de $P$. caseicolum constitue un argument intéressant en faveur de l'absorption et de l'accumulation possibles du calcium et du phosphore dans la croûte du fromage par l'intermédiaire de ce champignon.

Nous avons tenté de déterminer l'étendue de ce processus de " pompage " en suivant la variation des teneurs en calcium et en $\mathrm{Pi}$ 


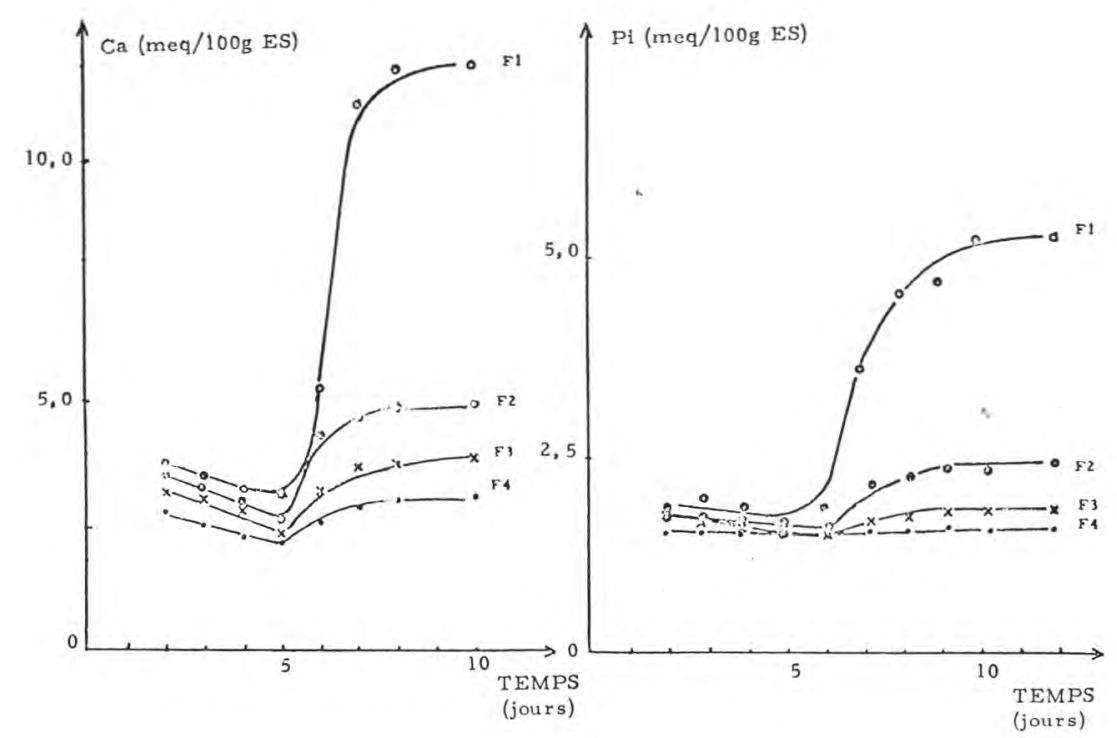

fig. 3

Variation des teneurs en $\mathrm{Ca}$ et en $\mathrm{Pi}$ au cours de l'affinage des fromages, en fonction de la profondeur de la fraction prélevée. F1 est la fraction la plus externe, F 4 est la fraction la plus interne.

dans des tranches de fromages horizontales de $3 \mathrm{~mm}$ d'épaisseur. Ces fractions sont notées de l'extérieur vers l'intérieur de F 1 à F 7 (soit une épaisseur totale de $21 \mathrm{~mm}$ ). Les résultats regroupés figure 3 permettent de montrer que l'accumulation du calcium et du phosphore se produit surtout dans la zone la plus externe (F 1), et à un degré moindre dans les zones (F 2) et (F 3). Au-delà de la zone (F 4), le processus d'accumulation se trouve considérablement amorti par suite de l'établissement d'un équilibre de diffusion et d'échange auquel sont associés vraisemblablement les ions $\mathrm{Na}^{+}$et $\mathrm{Cl}^{-}$provenant du salage superficiel du fromage. Il est probable aussi que cet effet dépende étroitement de la texture du milieu et de la répartition des molécules d'eau solvante.

Afin d'étayer notre hypothèse sur le rôle actif de la flore fongique dans l'accumulation de $\mathrm{Ca}^{++}$et de $\mathrm{Pi}$, nous avons essayé de comparer directement les résultats d'une accumulation par un processus dominant de diffusion, à ceux résultant d'une activité biologique.

A cette fin, un lot de 10 fromages est badigeonné à l'aide d'une solution antifongique de Pimaricine à $10 \mu \mathrm{g} / \mathrm{ml}$ pour inhiber la croissance du Penicillium, tandis qu'un deuxième lot identique au précédent est affiné normalement et sert de témoin. 


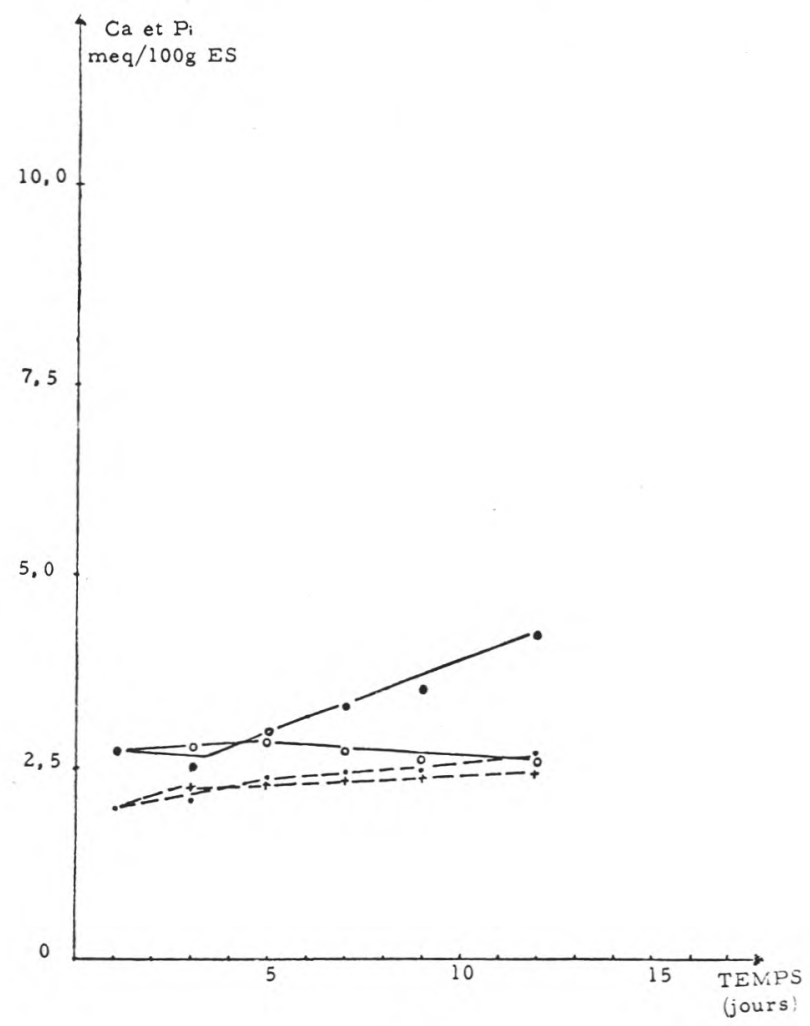

fig. 4

Etude de la variation des teneurs en calcium et en $\mathrm{Pi}$ dans la partie interne ( $\mathrm{CaO} \longrightarrow \mathrm{O}, \mathrm{Pi+ー} ー-+)$ et dans la partie externe ( $\mathrm{Ca} \bullet \bullet, \mathrm{Pi} \bullet--\bullet)$ des fromages traités à la Pimaricine.

Les teneurs en Ca et en Pi (meq/100 g d'extrait sec) sont déterminées comparativement et reportées figure 4 en fonction du temps d'affinage.

A l'intérieur, les variations des teneurs en $\mathrm{Ca}^{++}$et en $\mathrm{Pi}$ sont faibles ; quant à l'extérieur, il y a lieu de noter un accroissement quasi linéaire, mais peu important du calcium en fonction du temps. En tout état de cause, le processus de migration des ions est profondément perturbé et sans commune mesure avec le processus lié au développement du mycélium.

Si l'on compare ces résultats avec ceux de la figure 1, il apparaît une différence significative au niveau de la partie périphérique du 


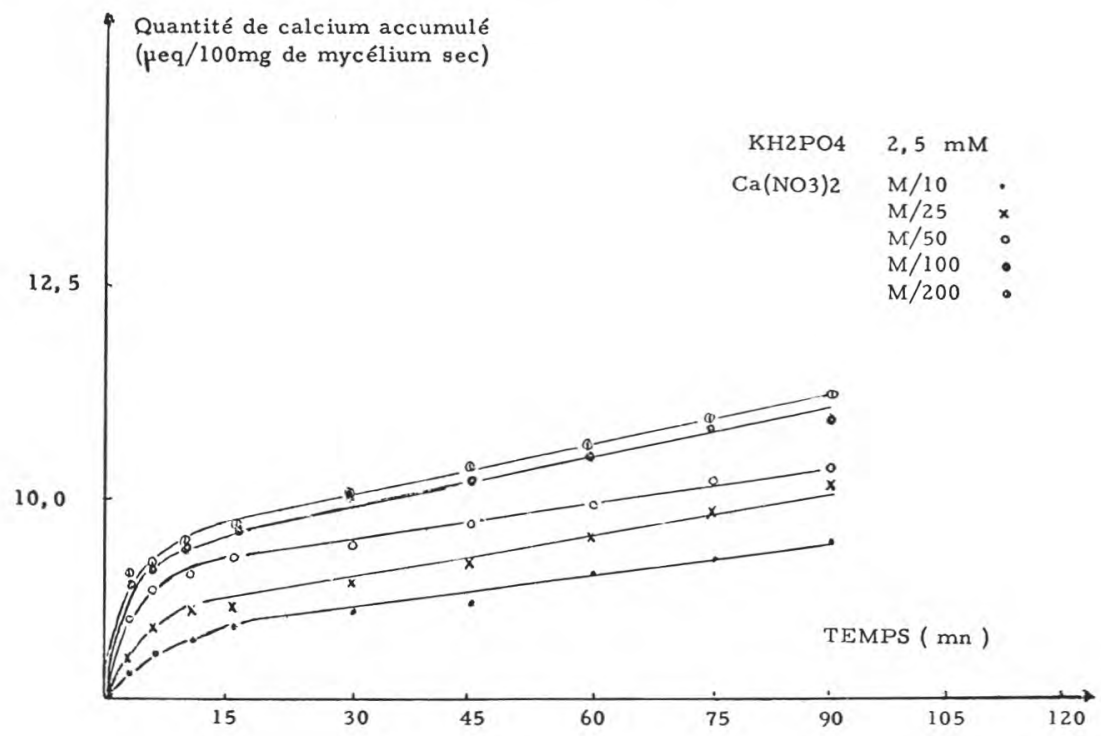

fig. 5

Accumulation du calcium dans le mycélium de $P$. caseicolum, cultivé en milieu liquide et incubé $90 \mathrm{mn}$ dans le milieu de Watson et Smith contenant des concentrations variables en $\mathrm{Ca}\left(\mathrm{NO}_{3}\right)_{2}$.

fromage, ce qui semble justifier notre hypothèse, le calcium pouvant être finalement engagé dans des formes ou combinaisons salines solubles ou peu solubles.

Il est dès lors tentant de conclure que la croûte du fromage est le siège d'une accumulation biologique du calcium et du phosphore qui complète efficacement les processus physico-chimiques de diffusion et d'échange.

\section{Cinétique de la pénétration du calcium dans le mycélium de « $P$. caseicolum » cultivé en milieu liquide}

a) Influence de la concentration en calcium

Nous avons reporté figure 5, les quantités de calcium accumulées dans le mycélium en fonction du temps ( $\mu$ eq de $\mathrm{Ca}^{++} / 100 \mathrm{mg}$ de mycélium sec) pour différentes concentrations en nitrate de calcium du milieu d'incubation (voir § II-5).

Il apparaît de manière très nette que le calcium s'accumule en fonction du temps dans le mycélium. Les quantités accumulées sont 


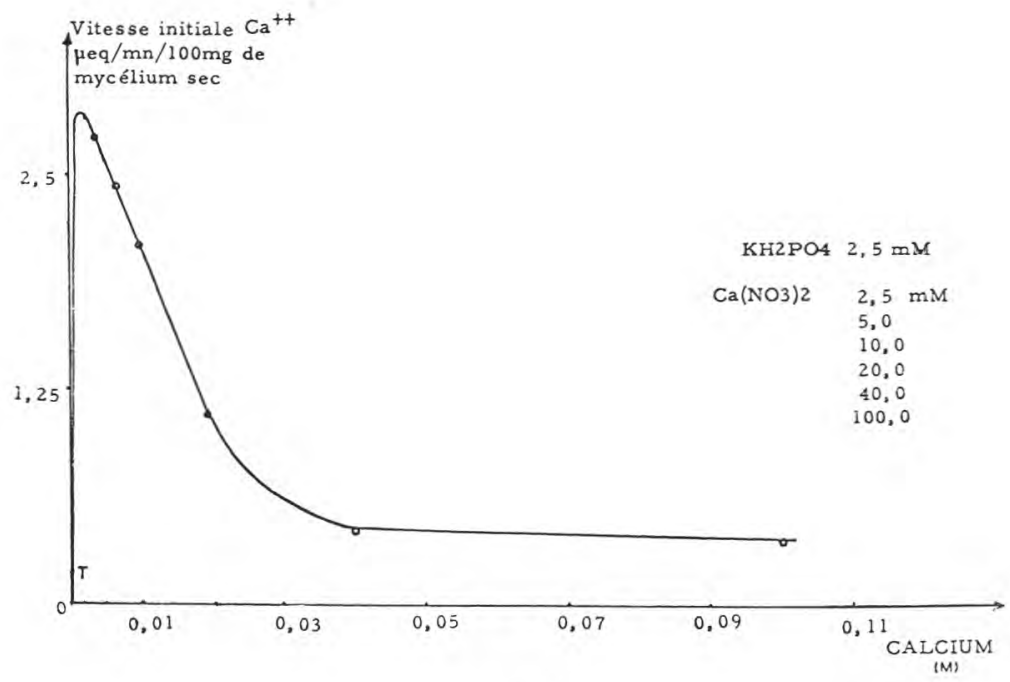

fig. 6

Vitesse initiale d'accumulation du calcium dans le mycélium de $P$. caseicolum, cultivé en milieu liquide et incubé $90 \mathrm{mn}$ dans le milieu de Watson et Smith contenant des concentrations variables en $\mathrm{Ca}\left(\mathrm{NO}_{3}\right)_{2}$. La lettre $\mathrm{T}$ se rapporte à la teneur initiale du mycélium en calcium.

d'autant plus grandes que les concentrations en nitrate de calcium tendent vers une valeur optimale voisine de 0,005 $\mathrm{M}$.

Ces résultats peuvent être exprimés différemment en portant les vitesses initiales de pénétration du calcium ( $\mu$ eq de $\mathrm{Ca}^{++} / \mathrm{mn} / 100 \mathrm{mg}$ de mycélium sec) en fonction de la concentration en calcium dans le milieu environnant (fig. 6). La courbe représentative fait apparaître trois phases apparemment distinctes dans le processus de transport. La première correspond à une activation du processus d'accumulation du calcium par le mycélium pour de faibles concentrations dans le milieu environnant en nitrate de calcium, inférieures à $M / 200$. Elle se traduit par une augmentation rapide des vitesses initiales de pénétration jusqu'à un optimum voisin de $2,9 \mu$ eq de $\mathrm{Ca}^{++} / \mathrm{mn} / 100 \mathrm{mg}$ de mycélium sec. La seconde qui ne se manifeste que pour des concentrations en nitrate de calcium supérieures à 0,005 M fait apparaître au contraire une influence inhibitrice se traduisant par une diminution progressive quasi linéaire de la vitesse de pénétration du calcium et qui peut s'interpréter par l'existence d'un processus proportionnel à la concentration en $\mathrm{Ca}^{++}$.

A partir d'une concentration en calcium voisine de $0,035 \mathrm{M}$, la vitesse initiale devient pratiquement constante $\left(0,42 \mu \mathrm{eq} \mathrm{Ca}^{++} / \mathrm{mn} /\right.$ $100 \mathrm{mg})$, elle ne varie plus malgré l'accroissement de la teneur en 


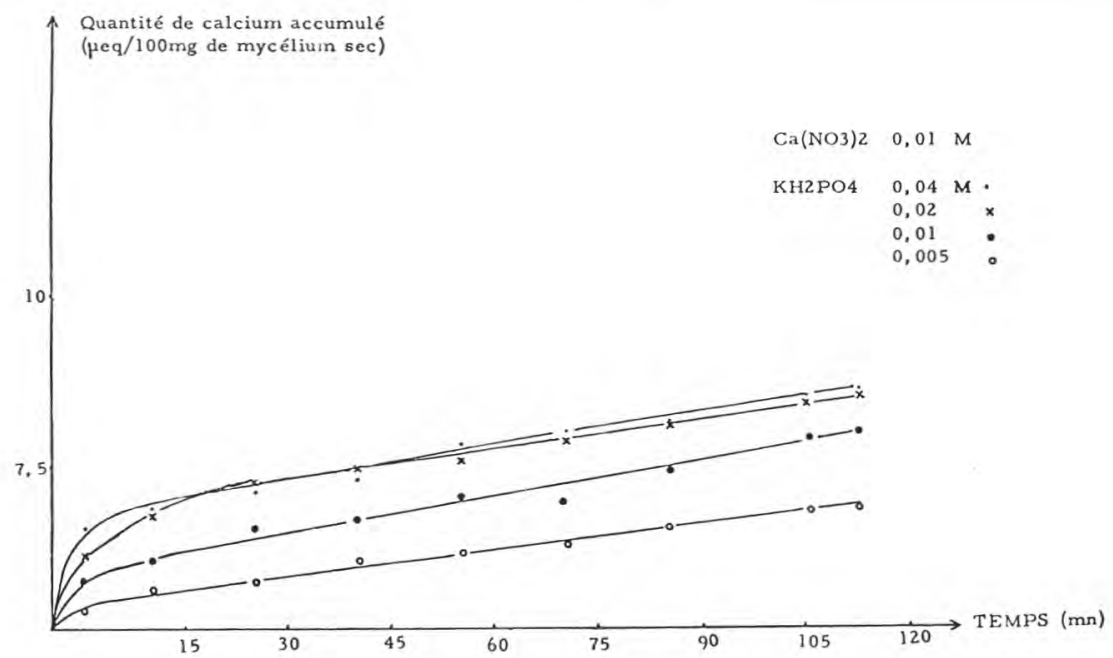

fig. 7

Accumulation du calcium dans le mycélium de $P$. caseicolum, cultivé en milieu liquide et incubé $90 \mathrm{mn}$ dans le milieu de Watson et Smith contenant des concentrations variables en $\mathrm{KH}_{2} \mathrm{PO} 4$.

calcium du milieu environnant. Cette valeur est d'ailleurs très voisine de celle que l'on observe pour le témoin $(0,21 \mu \mathrm{eq} / \mathrm{mn} / 100 \mathrm{mg})$ en l'absence de calcium ajouté au milieu d'incubation.

On peut interpréter aussi ces résultats en envisageant l'existence de deux étapes cinétiques différentes, la première $\left(0<\mathrm{Ca}^{++} \leqslant 0,005 \mathrm{M}\right)$ étant sous la dépendance d'un mécanisme d'entrée diffusionnel, tandis que la seconde $\left(0,005 \mathrm{M}<\mathrm{Ca}^{++}\right)$, en révélant l'intervention d'un transporteur intermédiaire, manifeste un processus de saturation qui se traduit par une diminution de la vitesse de pénétration, en fonction de l'accroissement de la concentration en $\mathrm{Ca}^{++}$du milieu environnant.

Lorsqu'on remplace le système lactate de potassium plus $\mathrm{Ca}\left(\mathrm{NO}_{3}\right)_{2}$, par le système équivalent, glucose plus $\mathrm{CaCl}_{2}$, on obtient des résultats très différents : la relation entre la vitesse initiale de pénétration et la concentration en $\mathrm{Ca}^{++}$dans le milieu devient anarchique. On peut alors penser que l'anion $\mathrm{Cl}^{-}$inhibe la pénétration du calcium, à moins que le remplacement du lactate n'ait pour effet de limiter l'énergie disponible nécessaire au fonctionnement du processus de transport.

b) Influence de la concentration en phosphate

Sur la figure 7 , nous avons reporté les quantités de calcium exprimées en $\mu$ eq de $\mathrm{Ca}^{++} / 100 \mathrm{mg}$ de mycélium sec en fonction du 


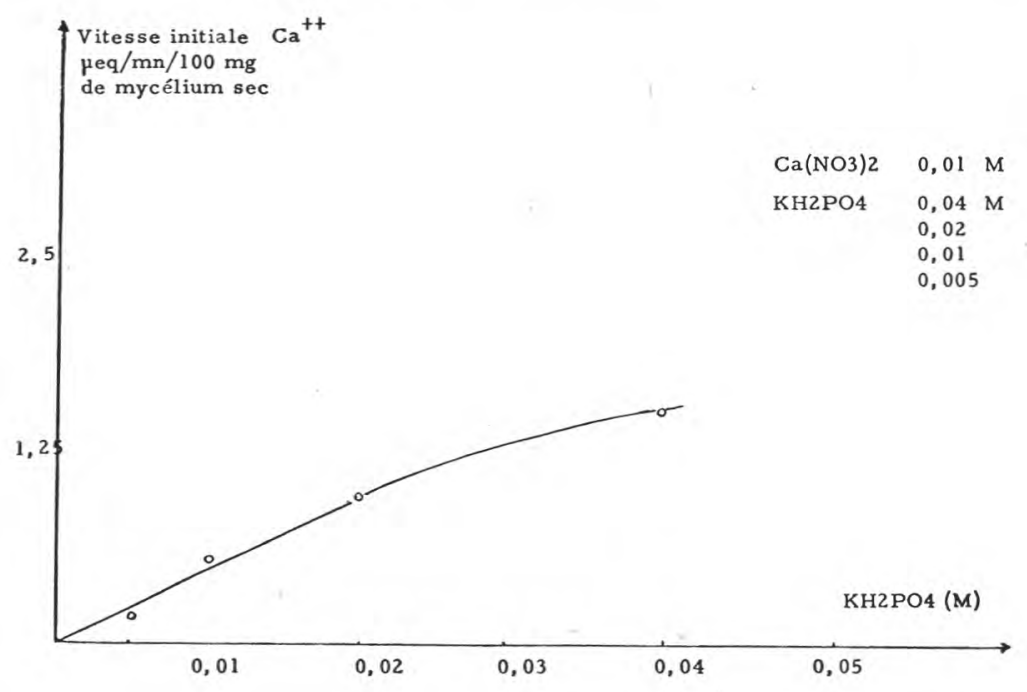

fig. 8

Vitesse initiale d'accumulation du calcium dans le mycélium de $P$. caseicolum, cultivé en milieu liquide et incubé $90 \mathrm{mn}$ dans le milieu de Watson et Smith contenant des concentrations variables en $\mathrm{KH}_{2} \mathrm{PO} 4$.

temps d'incubation (voir $\S$ II-5) pour des concentrations variables en $\mathrm{KH}_{2} \mathrm{PO} 4 \mathrm{du}$ milieu d'incubation comprises entre 0,005 et 0,04 M.

La vitesse initiale d'accumulation $\mathrm{du} \mathrm{Ca}^{++}$s'accroît pratiquement d'une manière quasi linéaire pour des concentrations en $\mathrm{Pi}$ comprises entre 0 et $0,002 \mathrm{M}$; ensuite, elle diminue progressivement et tend vers une valeur constante voisine de $1,5 \mu \mathrm{eq} \mathrm{de} \mathrm{Ca}^{++} / \mathrm{mn} / 100 \mathrm{mg}$ de mycélium sec lorsque la concentration en Pi atteint et dépasse 0,005 M (fig. 8).

Ces résultats tendent donc à confirmer l'idée que les phosphates sont transportés simultanément avec le calcium et que la pénétration de ces ions est sous la dépendance de processus de phosphorylation.

c) Influence de l'ADP et de l'ATP sur la pénétration et l'accumulation du calcium

Nous avons étudié l'influence de l'ADP $1 \mathrm{mM}$ et de l'ATP $1 \mathrm{mM}$ en présence de phosphates $\left(\mathrm{KH}_{2} \mathrm{PO} 42,5 \mathrm{mM}\right)$ et de lactate de potassium $10 \mathrm{mM}$ comme source énergétique, sur la vitesse initiale de pénétration du calcium (concentrations de $\mathrm{Ca}\left(\mathrm{NO}_{3}\right)_{2}$ comprises entre 0 et $0,4 \mathrm{M}$ ). 


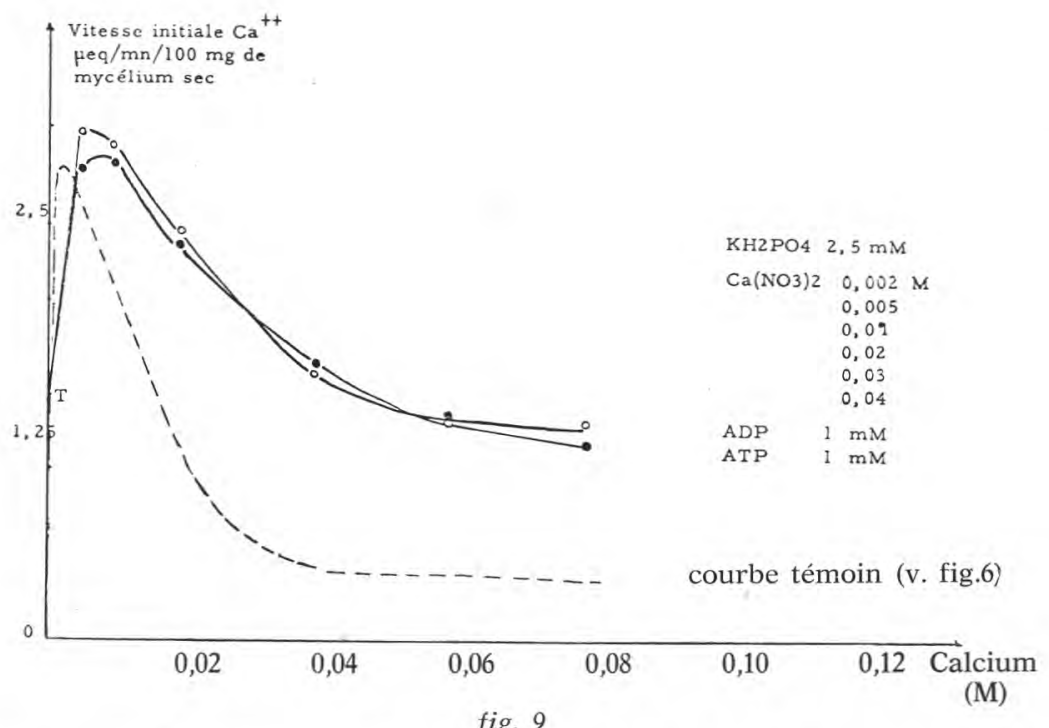

Vitesse initiale d'accumulation du calcium dans le mycélium de $P$. caseicolum, cultivé en milieu liquide et incubé $90 \mathrm{mn}$ dans le milieu de Watson et Smith contenant des concentrations variables en $\mathrm{Ca}\left(\mathrm{NO}_{3}\right)_{2}$ avec l'un ou l'autre des cofacteurs énergétiques ADP et ATP $1 \mathrm{mM}$. Comparaison avec la courbe de la figure 7 .

Les résultats, exprimés par les courbes de la figure 9, tendent à montrer que l'influence des 2 cofacteurs ADP et ATP, en présence d'une source d'énergie telle que le lactate, sont identiques.

D'autre part, tout se passe comme si pour des concentrations en lactate de calcium inférieures ou égales à 0,005 M, l'ADP et l'ATP étaient sans influence sur l'accroissement de la vitesse initiale de pénétration du calcium, ce qui tendrait à accréditer l'idée de l'existence d'un processus de pénétration par diffusion simple pour des concentrations en $\mathrm{Ca}^{++}$inférieures à $0,005 \mathrm{M}$. Cette première phase serait insensible à la fourniture d'énergie métabolique.

Par contre, la deuxième phase qui se manifeste pour des concentrations en calcium supérieures à $0,005 \mathrm{M}$, est nettement sensibilisée par l'ADP et l'ATP. La comparaison des courbes de la figure 6 et de la figure 9 permet de montrer que l'ADP et l'ATP ont pour effet de s'opposer à la diminution de la vitesse initiale du processus de pénétration consécutive à l'accroissement de la concentration en $\mathrm{Ca}^{++}$ dans le milieu environnant.

Pour des concentrations en $\mathrm{KH}_{2} \mathrm{PO} 42,5 \mathrm{mM}$ les courbes représentatives des vitesses initiales en présence d'ADP ou d'ATP $1 \mathrm{mM}$ 
sont pratiquement confondues et traduisent une diminution beaucoup moins rapide de la vitesse d'entrée de $\mathrm{Ca}^{++}$que dans le cas où I'ADP et l'ATP sont absents.

Ces résultats confirment bien l'importance de l'intervention de l'énergie métabolique dans ce processus et la tendance à faire pénétrer plus de calcium pour des concentrations en calcium du milieu environnant, supérieures à $0,005 \mathrm{M}$.

Il est intéressant, en outre, de remarquer que le comportement en présence d'ATP est identique à celui que l'on observe avec l'ADP, lorsque ce dernier est associé au catabolisme d'un substrat énergétique tel que le lactate.

\section{Cinétique de pénétration des phosphates}

Nous avons étudié l'incorporation de ${ }^{32} \mathrm{Pi}$ dans le mycélium de $P$. caseicolum pour une teneur constante en protéines $(10 \mathrm{mg})$, les concentrations en $\mathrm{KH}_{2} \mathrm{PO} 4$ du milieu environnant étant comprises entre 0 et $8 \mathrm{mM}$. Les résultats sont exprimés par les courbes de la figure 10. En présence de $\mathrm{Ca}\left(\mathrm{NO}_{3}\right)_{2}$ ajouté au milieu, la pénétration de ${ }^{32} \mathrm{Pi}$ se traduit par une accumulation rapide de la radioactivité dans le mycélium qui tend vers une limite proche de 0,007 $\mu$ eq de $\mathrm{Pi} / 100 \mathrm{mg}$ de mycélium sec pour des concentrations en Pi supérieures à $8 \mathrm{mM}$.

Pour des concentrations en $\mathrm{Ca}\left(\mathrm{NO}_{3}\right)_{2}(0,1 \mathrm{M}$ et $0,01 \mathrm{M})$ ajouté au milieu, la pénétration de ${ }^{32} \mathrm{Pi}$ est plus ou moins freinée. Cet effet pratiquement inexistant pour la concentration $0,01 \mathrm{M}$ devient notable pour une concentration en $\mathrm{Ca}\left(\mathrm{NO}_{3}\right)_{2} 0,1 \mathrm{M}$, ce qui tend bien à montrer l'existence d'une interdépendance entre le processus d'entrée du calcium et des phosphates.

\section{a) Influence de l'ADP et de l'ATP sur la pénétration} des phosphates

L'examen, d'une part des courbes de la figure 11 et la comparaison de cette dernière avec la courbe de la figure 10 , permettent de compléter les informations précédentes sur la pénétration des ions phosphates dans le mycélium de $P$. caseicolum. Pour une concentration constante en ATP $(1 \mathrm{mM})$, la vitesse de pénétration de ${ }^{32} \mathrm{Pi}$ (exprimée en $\mu$ eq $\mathrm{Pi} / \mathrm{mg}$ de mycélium/mn) est diminuée lorsque la concentration en $\mathrm{Ca}^{++}\left(\mathrm{Ca}\left(\mathrm{NO}_{3}\right)_{2}\right)$ du milieu s'accroît de $0,001 \mathrm{M}$ à $0,1 \mathrm{M}$.

Le rapport des vitesses atteint 1,55.

La même observation est valable pour l'ADP $1 \mathrm{mM}$, le rapport des vitesses atteignant dans ce cas 2,0. Toutefois, l'influence de l'ADP sur la vitesse de pénétration de $\mathrm{Pi}$ est plus faible, ce qui se traduit, à concentration égale en $\mathrm{Ca}^{++}$, par un effet d'inhibition plus fort du calcium sur la pénétration de $\mathrm{Pi}$. 


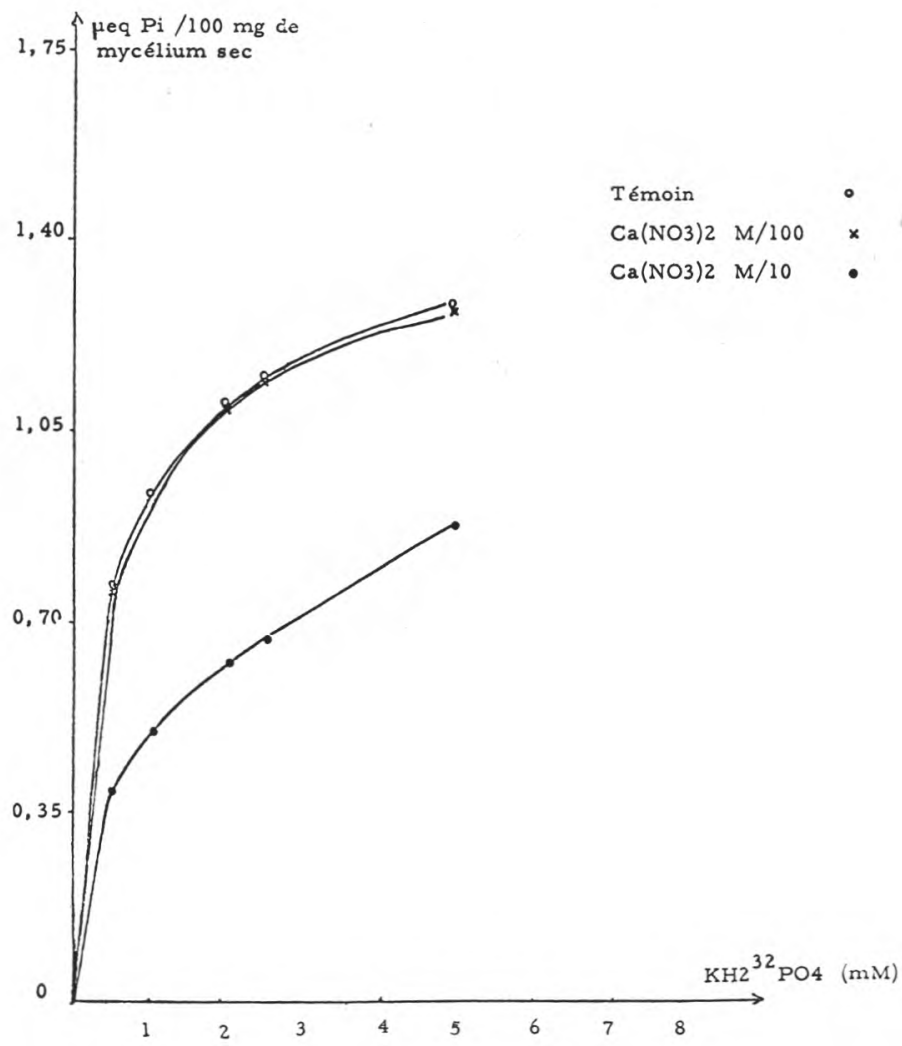

fig. 10

Accumulation de phosphate radioactif $(\mathrm{Pi})$ dans le mycélium de $P$. caseicolum, cultivé en milieu liquide et incubé $90 \mathrm{mn}$ dans le milieu de Watson et Smith contenant des concentrations variables en $\mathrm{Pi}$. L'expérience est conduite pour deux concentrations en calcium $(\mathrm{M} / 10$ et $\mathrm{M} / 100)$, en présence d'un témoin dépourvu de calcium et en l'absence d'ADP ou d'ATP.

D'autre part, pour des concentrations équivalentes en $\mathrm{Ca}^{++}$0,01 $\mathrm{M}$ ou $0,1 \mathrm{M}$, il est curieux d'observer que l'apport d'ATP $1 \mathrm{mM}$ ou d'ADP $1 \mathrm{mM}$, a pour effet de diminuer systématiquement la pénétration de $\mathrm{Pi}$ pour des concentrations en $\mathrm{KH}_{2} \mathrm{PO} 4$ comprises entre 0 et $2 \mathrm{mM}$. L'effet est plus accusé pour l'ADP que pour l'ATP, tout se passe comme si pour des concentrations en $\mathrm{Pi}$ de 0 à $2 \mathrm{mM}$, ces cofacteurs renforçaient l'inhibition due au $\mathrm{Ca}^{++}$.

Par contre, pour des concentrations en Pi supérieures à $2 \mathrm{mM}$, l'accroissement de la vitesse de pénétration par rapport au témoin 


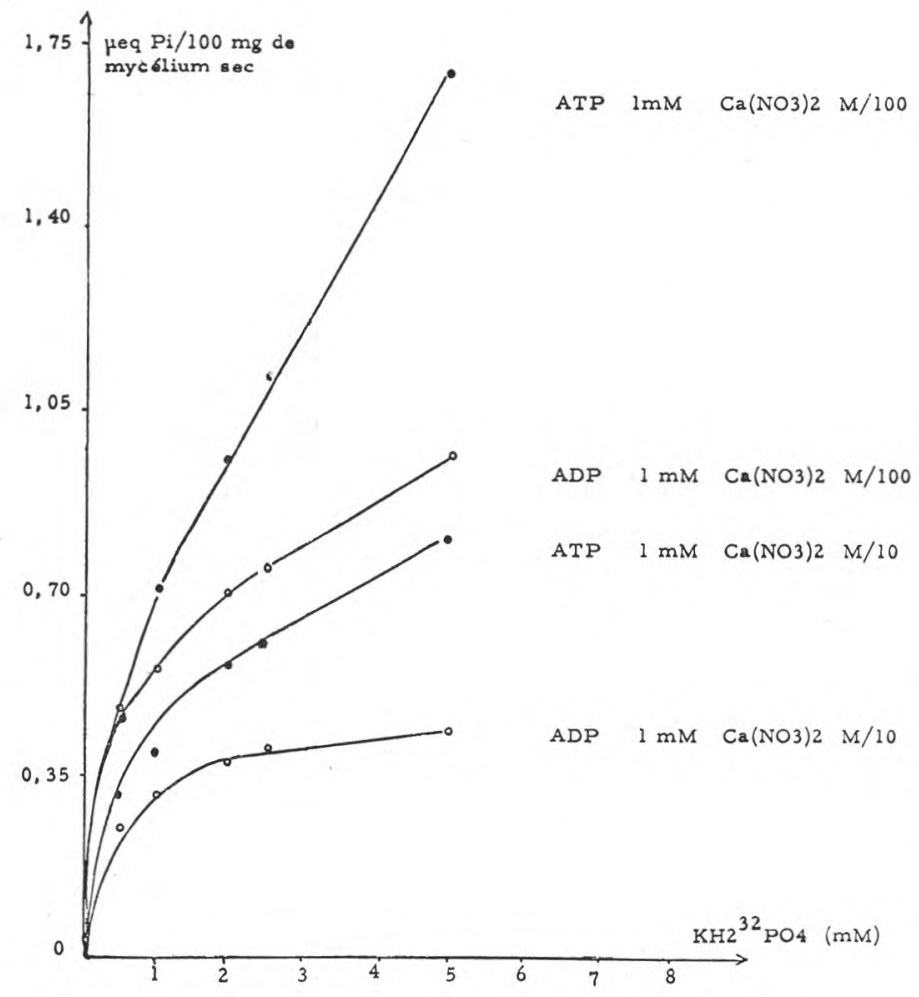

fig. 11

Accumulation de phosphate radioactif (Pi) dans le mycélium de $P$. caseicolum, cultivé en milieu liquide et incubé $90 \mathrm{mn}$ dans le milieu de Watson et Smith contenant des concentrations variables en $\mathrm{Pi}$. L'expérience est conduite pour deux concentrations en calcium $(\mathrm{M} / 10$ et $\mathrm{M} / 100)$, en présence d'ADP ou d'ATP $1 \mathrm{mM}$.

(sans ADP ou ATP), est d'autant plus net que la concentration en $\mathrm{Ca}^{++}$ est plus faible $\left(\mathrm{Ca}^{++} 0,01 \mathrm{M}\right)$ et que le cofacteur énergétique est l'ATP.

A noter enfin, d'après la comparaison des courbes des figures 9 et 11 que pour des concentrations en $\mathrm{Pi}$ supérieures à $2 \mathrm{mM}(2,5 \mathrm{mM})$ et des concentrations en $\mathrm{Ca}\left(\mathrm{NO}_{3}\right)_{2}, 0,01 \mathrm{M}$ ou $0,1 \mathrm{M}$, il semble exister une relation étroite entre les quantités de calcium et de $\mathrm{Pi}$ incorporés en présence d'ATP $1 \mathrm{mM}$. Toutefois, il est difficile d'exprimer pour le moment un rapport rigoureux, le nombre de déterminations étant insuffisant. 


\section{CONCLUSION}

L'étude de la distribution du calcium et des phosphates dans les fromages du type Camembert nous a permis de mettre en évidence un processus d'accumulation de ces ions au niveau de la croûte. Cette accumulation est la résultante de processus de diffusion et d'échange auxquels se superpose une activité biologique liée au développement superficiel de la moisissure $P$. caseicolum.

Le comportement des fromages en l'absence de flore fongique après inhibition par la Pimaricine, fait apparaître une différence sensible d'accumulation du $\mathrm{Ca}$ et du $\mathrm{Pi}$ au niveau de la croûte, comparativement aux fromages non traités.

Afin d'approfondir cette étude et de préciser les principaux paramètres qui conditionnent ce processus il nous a paru nécessaire de modéliser notre système.

Pour nous affranchir à la limite de la géométrie et des conditions de structure spécifique au fromage, nous avons transposé l'étude au niveau du mycélium de $P$. caseicolum, cultivé en milieu homogène.

Bien qu'il s'agisse d'une simplification très schématique du système fromage-mycélium, nous pensons cependant que qualitativement les résultats obtenus dans ces conditions tendent à confirmer la contribution directe du mycélium au déplacement des ions $\mathrm{Ca}^{++}$ et $\mathrm{Pi}$ des parties sous-jacentes du fromage vers la croûte. Calcium et phosphates pénètrent dans le mycélium selon un processus plus complexe qu'un simple transport par diffusion ou par échange. Les quantités cumulées sont d'autant plus importantes que les concentrations en nitrate de calcium tendent vers une valeur optimale voisine de $0,005 \mathrm{M}$.

Le processus de perméation présente deux phases distinctes: la première se traduit par un mécanisme diffusionnel, avec accroissement rapide de la vitesse initiale de pénétration proportionnel à la concentration en $\mathrm{Ca}^{++}$dans le milieu environnant, jusqu'à une valeur optimale de 2,9 ueq de $\mathrm{Ca}^{++} / \mathrm{mm} / 100 \mathrm{mg}$ de mycélium sec. $\mathrm{La}$ seconde ne se manifeste que pour des concentrations en $\mathrm{Ca}^{++}$supérieures à $0,005 \mathrm{M}$ et s'accompagne d'une diminution de vitesse, fonction de l'accroissement de la concentration en calcium. Ce comportement fait apparaître un mécanisme de saturation que l'on peut imputer à l'intervention d'un transporteur intermédiaire.

Pour des concentrations en Pi comprises entre 0 et $0,02 \mathrm{M}$ la vitesse initiale d'accumulation de $\mathrm{Ca}^{++} \mathrm{s}^{\prime}$ accroît linéairement, ensuite

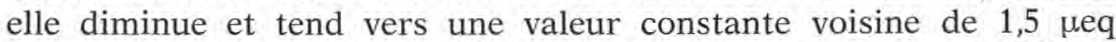
de $\mathrm{Ca}^{++} / \mathrm{mn} / 100 \mathrm{mg}$ de mycélium sec. Dans ces conditions l'ADP ou l'ATP (1 mM) ajoutés au système, pour une concentration en $\mathrm{Ca}^{++} \mathrm{du}$ milieu $0,005 \mathrm{M}$, sont sans influence sur la vitesse initiale de pénétration du cation. Par contre pour des concentrations en calcium supérieures à $0,005 \mathrm{M}$ le processus est nettement sensibilisé par cet apport 
énergétique; tout se passe comme si en présence d'ADP ou d'ATP davantage de $\mathrm{Ca}^{++}$pénétrait dans le mycélium.

Il n'en demeure pas moins qu'au cours de ce processus la vitesse de pénétration du calcium décroît lorsque la concentration en $\mathrm{Ca}^{++}$du milieu environnant augmente. Inversement la pénétration des ions Pi est freinée par l'accroissement de la concentration en $\mathrm{Ca}^{+}$ du milieu environnant, ce qui montre bien l'existence d'une interdépendance des processus d'entrée de $\mathrm{Ca}^{++}$et de $\mathrm{Pi}$.

Notons aussi que pour des concentrations respectives en $\mathrm{Pi}$ supérieures à $0,002 \mathrm{M}$ et en $\mathrm{Ca}^{++}$comprises entre $0,01 \mathrm{M}$ et $0,1 \mathrm{M}$ il semble exister une relation étroite entre les quantités de $\mathrm{Ca}^{++}$et de $\mathrm{Pi}$ incorporées en présence d'ATP.

Enfin il est intéressant de relever que les ions $\mathrm{Cl}^{-}$inhibent la pénétration du calcium et que le lactate constitue un excellent substrat énergétique pour le mycélium, équivalent au glucose.

Nous sommes donc tentés momentanément de conclure que $P$. caseicolum est capable d'accumuler du calcium et que les processus de pénétration de ce cation et des ions $\mathrm{Pi}$ sont étroitement liés. Il apparaît en outre que pour des concentrations du milieu en $\mathrm{Ca}^{++}$supérieures ou égales à $0,005 \mathrm{M}$ le processus est sensible à l'apport d'énergie métabolique. Cet ensemble d'observations permet finalement de penser que l'accumulation du calcium est sous la dépendance d'un transporteur, toutefois il est difficile pour le moment

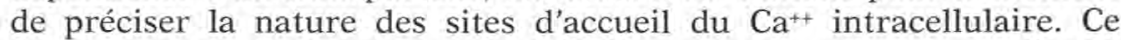
dernier aspect intéresse tout particulièrement le mécanisme d'accumulation du calcium au niveau de la croûte du fromage; des travaux en cours doivent nous permettre prochainement de préciser ce problème.

\section{R és u m é}

Au cours de l'affinage des fromages du type Camembert, la partie périphérique de ceux-ci est le siège d'un accroissement significatif des teneurs en calcium et en phosphore. Le processus d'accumulation se développe parallèlement à la croissance exponentielle de $P$. caseicolum. L'allure de ce processus, ainsi que les quantités de calcium et de phosphore rassemblées tendent à montrer que ces deux éléments sont soumis en partie à un mécanisme d'accumulation de nature biologique. Des observations sur le comportement de la moisissure en milieu liquide viennent renforcer cette hypothèse. Dans ces conditions, nous avons pu montrer que les ions phosphates $\left(\mathrm{H}_{2} \mathrm{PO} 4^{-}\right)$ facilitent la pénétration du calcium jusqu'à la concentration de $0,05 \mathrm{M}$, au-delà de laquelle le système présente un phénomène de saturation. L'apport d'ADP ou d'ATP dans ce milieu se traduit par une stimulation de la pénétration du calcium lorsque la concentration en $\mathrm{Ca}^{++}$environnante est supérieure à $0,005 \mathrm{M}$. De même l'entrée des phosphates pour des concentrations en $\mathrm{Pi}$ supérieures à $2 \mathrm{mM}$ est d'autant plus 
importante que la concentration en $\mathrm{Ca}^{++}$est plus faible et que le cofacteur ajouté est l'ATP.

\section{S u $\mathrm{m} m$ a ry}

During the ripening of Camembert cheese, its external part shows a significant increase of its calcium and phosphorus concentrations. The process develops simultaneously with the exponential growth of $P$. caseicolum. The aspect of the process, just as the quantities of calcium and phosphorus accumulated lead us to think that they are both to a certain extent subjected to a biological accumulating mechanism.

Some observations made on the mould grown in a liquid medium merely strenghten this hypothesis. Under these conditions, we have been able to show that the phosphate ions $\left(\mathrm{H}_{2} \mathrm{PO}^{-}\right)$make the penetration of calcium easier in so far as its concentration does not exceed $0,005 \mathrm{M}$.

The system reaches saturation point with a higher concentration.

The adjunction of ADP or ATP to the medium has a stimulating effect on the $\mathrm{Ca}^{++}$penetration, when the $\mathrm{Ca}^{++}$concentration remains within a $0,005 \mathrm{M}$ to $0,12 \mathrm{M}$ limit. In the same way, the intake of phosphates for $\mathrm{Pi}$ concentrations higher than $2 \mathrm{mM}$, is all the more important as the $\mathrm{Ca}^{++}$concentrations are smaller, and the added cofactor is ATP.

Reçu pour publication en mars 1978

\section{Bibliographie}

[1] LenOIR (J.), ChOIsY (C.) (1971), - Le Lait, 503-504, 138-157.

[2] Lenoir (J.), Auberger (B.) (1977). - Le Lait, 563-564, 164-183.

[3] Lamberet (G.), Lenoir (J.) (1972). - Le Lait, 513-514, 175-192.

[4] Stan (H. J.), Podzuweit (T.) (1972), - Chemie Milch Techn. der Lebensmittel, $1,161-168$.

[5] Dijies (L.), Morfaux (J. N.) (1968), - Ann. Techn. Agric, 17, 4, 355-377.

[6] LiRillo (V. P.) (1961). - Ann. Rev. Microbiol., 15, 197-218.

[7] KePES (A.) (1960). - Biochim. Biophys. Acta, 40, 70-84.

[8] Jennings (D.), Hooper (D. C.), Rothstein (A.) (1958). - J. Gen. Physio., 11, 1019-1026.

[9] Schornen (O.), Borst-Pauwels (G. W. F.) (1967). - Biochim. Biophys. Acta, $15,785-799$.

[10] Silver (S.) (1969). - Proc. Nat. Acad. Sci. US, 62, 3, 764-771.

[11] Vasington (F. D.), Murphy (J. V.) (1962). - J. Biol. Chem., 237, 8, 2670-2677.

[12] Goodman (J.), Rothstein (A.) (1957). - J. Gen. Physiol., 40, 915-923.

[13] Bartlett (J.) (1959). - J. Biol. Chem., 234, 466.

[14] Watson (K.), Sмith (J. E.) (1968), - J. Bacteriol., 96, 1546-1550.

[15] Lin (W.), Hanson (J. B.) (1974). - Plant Physiol., 54, 250-256.

[16] WiLkins (P. O.) (1967), - J. of Bacteriol.. 93, 5, 1565-1570. 